\title{
ARTICLE
}

Minimal resdiual disease

\section{Standardized next-generation sequencing of immunoglobulin and T-cell receptor gene recombinations for MRD marker identification in acute lymphoblastic leukaemia; a EuroClonality-NGS validation study}

\author{
Monika Brüggemann ${ }^{1} \cdot$ Michaela Kotrová ${ }^{1,2} \cdot$ Henrik Knecht $^{1} \cdot$ Jack Bartram $^{3} \cdot$ Myriam Boudjogrha $^{4}$. \\ Vojtech Bystry ${ }^{5}$ - Grazia Fazio $\mathbb{D}^{6}$ - Eva Froňková ${ }^{2} \cdot$ Mathieu Giraud $\mathbb{D}^{7}$ - Andrea Grioni ${ }^{6}$. Jeremy Hancock ${ }^{8}$. \\ Dietrich Herrmann ${ }^{1} \cdot$ Cristina Jiménez $^{9} \cdot$ Adam Krejci $^{5}$ - John Moppett $\mathbb{1}^{10} \cdot$ Tomas Reigl $^{5} \cdot$ Mikael Salson $^{7}$. \\ Blanca Scheijen ${ }^{11}$ - Martin Schwarz ${ }^{1}$ - Simona Songia ${ }^{6}$. Michael Svaton ${ }^{2} \cdot$ Jacques J. M. van Dongen $^{12}$. \\ Patrick Villarese $^{13}$. Stephanie Wakeman ${ }^{8}$ - Gary Wright ${ }^{3}$ - Giovanni Cazzaniga ${ }^{6}$ - Frédéric Davi ${ }^{4}$. \\ Ramón García-Sanz ${ }^{9}$ David Gonzalez ${ }^{14}$ - Patricia J. T. A. Groenen ${ }^{11} \cdot$ Michael Hummel $^{15}$ - Elizabeth A. Macintyre ${ }^{13}$.

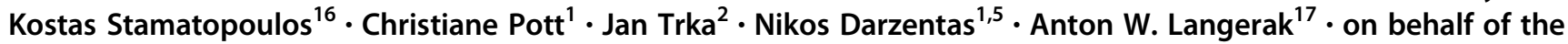 \\ EuroClonality-NGS working group
}

Received: 15 January 2019 / Accepted: 20 February 2019 / Published online: 26 June 2019

(c) The Author(s) 2019. This article is published with open access

\begin{abstract}
Amplicon-based next-generation sequencing (NGS) of immunoglobulin (IG) and T-cell receptor (TR) gene rearrangements for clonality assessment, marker identification and quantification of minimal residual disease (MRD) in lymphoid neoplasms has been the focus of intense research, development and application. However, standardization and validation in a scientifically controlled multicentre setting is still lacking. Therefore, IG/TR assay development and design, including bioinformatics, was performed within the EuroClonality-NGS working group and validated for MRD marker identification in acute lymphoblastic leukaemia (ALL). Five EuroMRD ALL reference laboratories performed IG/TR NGS in 50 diagnostic ALL samples, and compared results with those generated through routine IG/TR Sanger sequencing. A central polytarget quality control (cPT-QC) was used to monitor primer performance, and a central in-tube quality control (cIT-QC) was spiked into each sample as a library-specific quality control and calibrator. NGS identified 259 (average 5.2/sample, range 0-14) clonal sequences vs. Sanger-sequencing 248 (average 5.0/sample, range 0-14). NGS primers covered possible IG/TR rearrangement types more completely compared with local multiplex PCR sets and enabled sequencing of bi-allelic rearrangements and weak PCR products. The cPT-QC showed high reproducibility across all laboratories. These validated and reproducible quality-controlled EuroClonality-NGS assays can be used for standardized NGS-based identification of IG/TR markers in lymphoid malignancies.
\end{abstract}

These authors contributed equally: Monika Brüggemann, Michaela Kotrová

Supplementary information The online version of this article (https:// doi.org/10.1038/s41375-019-0496-7) contains supplementary material, which is available to authorized users.

Anton W. Langerak

a.langerak@erasmusmc.nl

Extended author information available on the last page of the article.

\section{Introduction}

Specific antigen recognition by cells of the adaptive immune system (B cells, T cells) is mediated through receptors (immunoglobulin, IG, and T-cell receptor, TR) that are uniquely formed during immune development in bone marrow and thymus, respectively. Through recombination of IG/TR loci a diverse (polyclonal) repertoire of unique IG/TR receptors is created. In certain autoimmune diseases this repertoire is skewed (oligoclonal), whereas in lymphoid malignancies receptors are largely identical (monoclonal) [1-7]. IG/TR rearrangements thus form 
unique genetic biomarkers (molecular signatures) for studying immune cells for clinical, diagnostic and research applications [8-11]. Classically, methods for immunogenetic analysis mostly concern fragment analysis and Sangerbased sequencing. The introduction of NGS makes deeper analysis of IG/TR rearrangements possible, with impact on the main immunogenetic applications: clonality assessment, MRD detection, repertoire analysis [12-29].

The EuroClonality-NGS working group (euroclonalityngs.org; Supplementary Figure 1) has ample expertise in development, standardization and validation of IG/TR assays, to address the challenges in the translational research towards clinical application.

Here we report on the development and standardization (see also accompanying manuscript by Knecht et al. [30]) of novel amplicon-based IG/TR NGS assays between September 2012 and October 2017, via a total of 14 international coordination and evaluation meetings (Supplementary Table 1). This study focuses on IG/TR marker identification in lymphoid malignancies for subsequent MRD analysis, and their multicentre validation in acute lymphoblastic leukaemia (ALL). Assay optimizations and modifications for other applications of IG/TR NGS are partly still ongoing and will be reported in separate publications.

\section{Materials and methods}

\section{General concept of assay design}

With the objective of developing a universal ampliconbased NGS approach for IG/TR sequence analysis at the DNA level, applicable in all lymphoid malignancies, assays for multiple IG/TR loci were designed: IG heavy (IGH), IG kappa (IGK), TR beta (TRB), TR gamma (TRG) and TR delta (TRD), including complete and incomplete rearrangements whenever applicable. IG lambda (IGL) was excluded due to its limited complementarity to other IG loci and its reduced diversity. TR alpha (TRA) was excluded due to its high complexity, severely hampering a reasonable multiplex PCR approach at the DNA level.

The IGH locus is rearranged in two steps. After initial coupling of a single IGHD gene to an IGHJ gene, an IGHV gene is joined to the incomplete IGHD-IGHJ rearrangement, resulting in a complete IGHV-IGHJ rearrangement. For amplification of complete IGH rearrangements, primers located in the FR1, FR2 and FR3 regions were designed, but here we only discuss the FR1 assay for marker identification in ALL (for application of IGH-VJ-FR3 assay in clonality testing see accompanying manuscript by Scheijen et al. [31]). IGHD-IGHJ rearrangements were amplified in a separate multiplex PCR reaction. The IGK light chain locus is composed of functional IGKV and IGKJ genes, as well as the so-called kappa deleting element (Kde) that can rearrange to IGKV genes, or to a recombination signal sequence (RSS) in the IGKJ-IGKC intron, leading to functional inactivation of the IGK allele. The IGKV forward primers were designed to be used in combination with IGKJ and Kde reverse primers in one multiplex reaction, whereas a second PCR was developed for the forward intron RSS and reverse Kde primers.

The TRB locus also features a two-step process with initial formation of incomplete TRBD-TRBJ rearrangements followed by complete TRBV-TRBJ rearrangements. Incomplete and complete TRB rearrangements are detected in two separate multiplex PCR reactions. As TRG locus rearrangements are one-step $\mathrm{VJ}$ recombinations involving a limited number of TRGV and TRGJ genes, a single multiplex assay could be developed. Finally, in the TRD locus, complete VJ rearrangements are preceded by DD, VD and DJ rearrangements. In addition, certain TRAV genes can rearrange to both TRDJ and TRAJ, whereas TRDV-TRAJ rearrangements, usually involving TRAJ29, can also occur. All of these rearrangements were designed to be amplified in one multiplex PCR assay.

Both the design and further testing were coordinated by the respective 'Target' network leaders: IGH-VJ by C. Pott, Kiel and R. Garcia Sanz, Salamanca; IGH-DJ by F. Davi, Paris and K. Stamatopoulos, Thessaloniki; IGK-V/intronIGKJ/Kde by P.J.T.A. Groenen, Nijmegen and A.W. Langerak, Rotterdam; TRB by M. Brüggemann, Kiel and M. Hummel, Berlin; TRG by G. Cazzaniga, Monza and J.J.M. van Dongen, Leiden; and TRD by E. Macintyre, Paris. Initial testing of each assay was performed by 2-3 experienced laboratories per target and final assays were validated for IG/TR marker identification in ALL in a multicentre setting. In addition, central quality control procedures were developed to monitor assay performance.

The bioinformatic platform ARResT/Interrogate [32], developed from the ground-up within the EuroClonalityNGS to assist with its multi-faceted activities, was further adapted for this study as described below.

\section{Primer design and technical validation of primer performance}

Primers were designed to be gene-specific, but in case of allelic variants, degenerate primers were designed to avoid differential annealing in individuals with different allelic variants. For the same reason, single mismatches in the middle or at the $5^{\prime}$-end of the primer were accepted.

Primer3 [33], Primer Digital (PrimerDigital Ltd, Helsinki, Finland) MFEprimer-2.0 [34] and Oligo (Molecular Biology Insights, Inc., Colorado, USA) were used for checking primer specificity and multiplexing. Common primer design criteria were followed for all loci: 
primer melting temperature $57-63{ }^{\circ} \mathrm{C}$; comparable size of final amplicon; primer length $20-24 \mathrm{nt}$; avoidance of primer dimers; minimal distance of $3^{\prime}$ primer end to the junctional region of, preferably, $>10-15$ bp to avoid false-negativity for rearrangements with larger nucleotide deletions from the germline sequence; avoidance of regions with known single nucleotide polymorphisms to allow identical primer annealing for all alleles of the respective $\mathrm{V}, \mathrm{D}$ or $\mathrm{J}$ genes; targeting of, preferably, all $\mathrm{V}, \mathrm{D}$ and $\mathrm{J}$ genes known to be rearranged plus the intronRSS and Kde regions for IGK.

Following in silico design, primers were first tested in monoplex and multiplex reactions using primary patient samples or cell lines with defined rearrangements. In occasional cases where no such samples were available, healthy tonsil or mononuclear DNA samples were employed. Oligoclonal template pools were then created from mixtures of rearranged cell lines and diagnostic samples with defined rearrangements covering many different V, D and/or J genes. Alternatively, for some loci, plasmid pools were produced, covering as many different rearrangements as possible. These multi-target pools allowed finetuning of reaction conditions and/or primer concentrations to assess comparable amplification efficiencies. This iterative process of testing also led to a reduction of primers if these appeared redundant. Further multicentre testing was performed with a limited number of monoclonal and poly/ oligoclonal samples on different sequencing platforms, which allowed assessment of robustness of the primer mixes and protocols.

As assays were designed with the aim to be platformindependent, a two-step PCR was employed, that enabled switching of sequencing adaptors and to reduce the total number of primers even if many barcodes are necessary. Also, maximal amplicon lengths were defined with respect to the possible maximal sequencing read lengths of current sequencers. PCR conditions were optimized with the aim to find optimal conditions common for all reactions, thus allowing for parallel library preparation. Various numbers of PCR cycles in 1st and 2nd PCR, different polymerases and several library purification methods were tested and compared.

\section{Multicentre validation of assays for MRD marker identification in ALL}

Five experienced laboratories tested the robustness and applicability of the optimized assays for NGS-based IG/TR marker identification in ALL in comparison to standard techniques. All laboratories (Bristol/London, Paris, Monza, Prague and Kiel) are members of the EuroMRD consortium and reference laboratories for ALL MRD analysis. Each of them performed NGS-based IG/TR MRD marker identification in 10 patients with B- or T-lineage ALL. A central standard operating procedure was strictly followed. The study was executed using the Illumina MiSeq $(2 \times 250 \mathrm{bp}$ v2 kit). NGS analyses were performed fully in parallel to conventional PCR plus Sanger sequencing of clonal products following standard guidelines [11]. For a part of the cases with unexplained discrepant results between the two methods, allele-specific PCR assays (either for digital droplet PCR or real-time quantitative PCR) were designed to clarify if the respective clonal rearrangement represented the leukaemic bulk. EuroMRD guidelines were used to design and interpret allele-specific PCR assays [35, 36].

\section{Results}

\section{Primer design and technical validation of primer performance}

Based on the results of the testing and validation phases (Supplementary Table 2), the final IG/TR primer mixes consisted of eight tubes with 92 forward and 30 reverse primers, 15 of the latter being used in pairs of different tubes (Supplementary Table 3). Primer positions and sequences are presented in Fig. 1.

\section{Implementation of quality control procedures}

Quality control of robust amplification, library preparation and sequencing are of utmost importance for these complex assays. Different primers need to work under the same reaction conditions, while additional variability can be introduced by sample characteristics and sequencing. Primer performance must be monitored longitudinally, and for the exact estimation of clonal abundance it is important to correct for the number of sequencing reads per input molecule.

To address these issues, we established and validated two types of quality control procedures: (i) a 'central in-tube quality control' (cIT-QC) spiked to each tube as library control and calibrator, and (ii) a 'central poly-target quality control' (cPT-QC), or run control, to monitor general primer performance and sequencing.

To compose the cIT-QC, IG/TR rearrangements of many human lymphoid cell lines were comprehensively characterized by amplicon- and capture-based NGS and Sanger sequencing. Nine cell lines were selected to form the cITQC with at least three different clonal rearrangements for each of the eight PCR tubes, totalling 24 rearrangements. The current design requires an equal number of cell line DNA copies to be spiked into each tube, as described below.

For the cPT-QC a mixture of different lymphoid specimens was considered to cover the whole IG/TR repertoire 

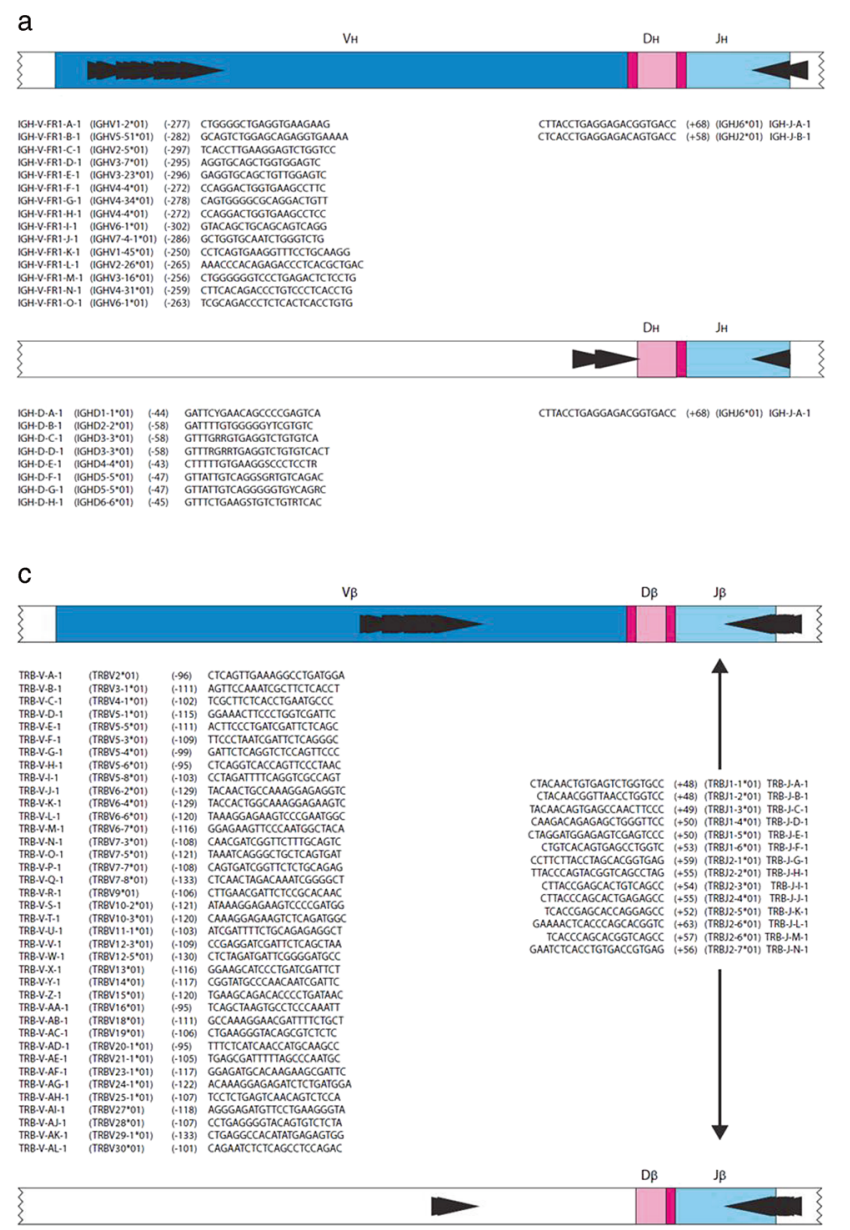

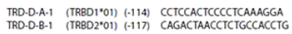

Fig. 1 Schematic diagrams of rearrangements and primer sets. a Schematic diagrams of IGHV-IGHJ and IGHD-IGHJ rearrangements. The relative position of the $\mathrm{VH}$ family primers, $\mathrm{DH}$ family primers and consensus $\mathrm{JH}$ primers is given according to their most $5^{\prime}$ nucleotide upstream (-) or downstream (+) of the involved RSS. b Schematic diagrams of IGKV-IGKJ rearrangement and the two types of Kde rearrangements (V-Kde and intronRSS-Kde). The relative position of the IGKV, IGKJ, Kde, and intronRSS (INTR) primers is given according to their most $5^{\prime}$ nucleotide upstream $(-)$ or downstream $(+)$ of the involved RSS. c Schematic diagrams of TRBV-TRBJ rearrangement and TRBD-TRBJ rearrangement. The relative position of the TRBV family primers, TRBD primers and the TRBJ primers is given

more comprehensively. To this end we produced material consisting of equal ratios of DNA from peripheral blood mononuclear cells (MNCs), thymus and tonsil. For more details see accompanying manuscript by Knecht et al. [30].

\section{Laboratory protocol}

Primers were tailed with universal and T7-linker sequences, and divided over eight tubes (IGH-VJ, IGH-DJ, IGK-VJKde, intron-Kde, TRB-VJ, TRB-DJ, TRG, TRD). The PCR b

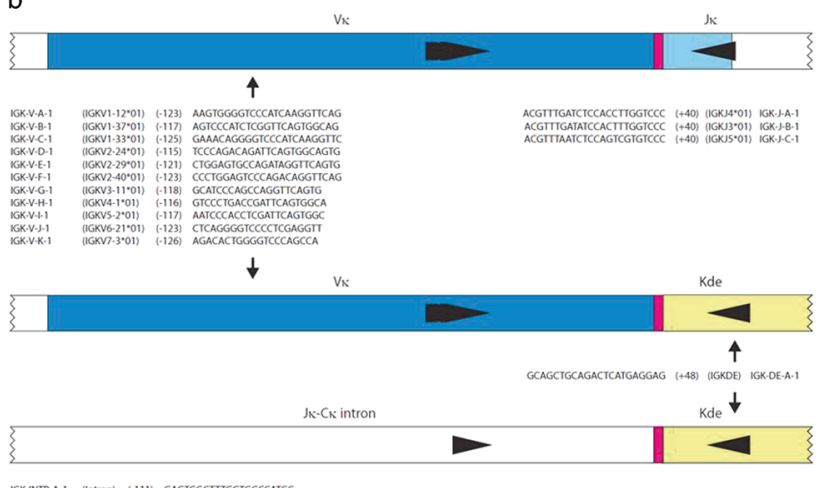

d
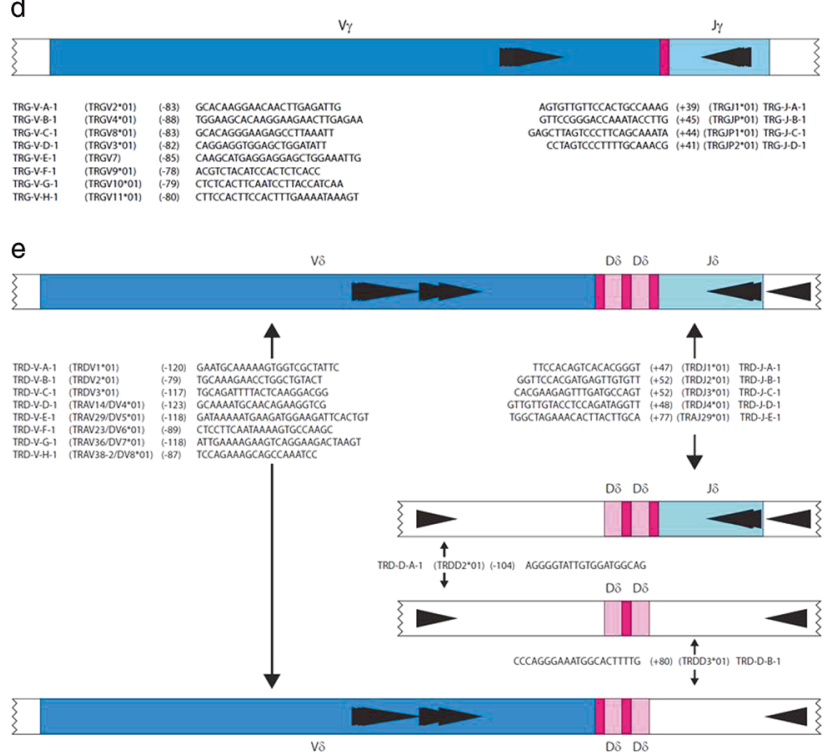

according to their most 5'nucleotide upstream (-) or downstream (+) of the involved RSS. d Schematic diagrams of TRGV-TRGJ rearrangement and the relative position of the TRGV and TRGJ primers. The relative position of the TRGV primers and the TRGJ primers is given according to their most 5 ' nucleotide upstream (-) or downstream $(+)$ of the involved RSS. e Schematic diagram of TRDVTRDJ,TRDD-TRDJ, TRDD-TRDD, and TRDV-TRDD, TRDVTRAJ29 rearrangements, showing the positioning of TRDV, TRDJ, TRDD, and TRAJ29 primers, all combined in a single tube. The relative position of the TRDV, TRDD, and TRDJ primers is indicated according to their most 50 nucleotides upstream $(-)$ or downstream (+) of the involved RSS

protocol is summarized in Table 1. Sequencing libraries were prepared via a two-step PCR, each using a final reaction volume of $50 \mu \mathrm{l}$ with $100 \mathrm{ng}$ diagnostic DNA and $10 \mathrm{ng}$ of polyclonal DNA. For the cIT-QC, 40 cell equivalents of the nine different cell lines were spiked into all samples (see accompanying manuscript by Knecht et al. [30]). $\mathrm{MgCl}_{2}$ was intended to be used at a final concentration of $1.5 \mathrm{mM}$, but needed optimization for some tubes. Therefore, master-mixes for the 1st PCR were tube-specific, but the temperature profile was uniform for all tubes. 


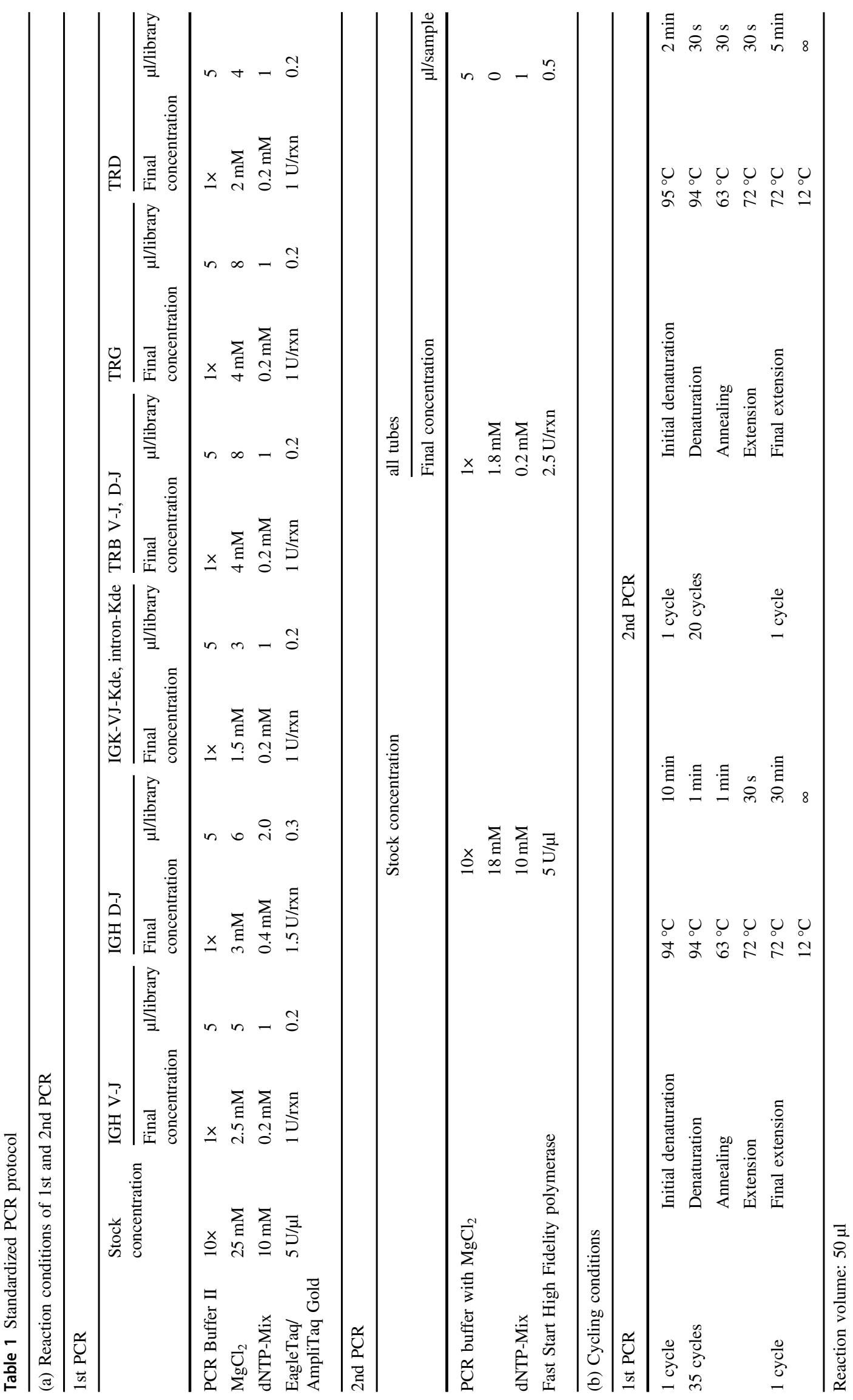


Table 2 Mean size of PCR products after the 2nd PCR (containing the Illumina sequencing adaptors and barcodes)

\begin{tabular}{ll}
\hline Gene & Amplicon length (bp) \\
\hline TRB-VJ & $309-407$ \\
TRB-DJ & $300-408$ \\
TRG & $256-360$ \\
TRD & $309-450$ \\
IGH-VJ & $484-681$ \\
IGH-DJ & $266-358$ \\
IGK-VJ-Kde & $296-384$ \\
intron-Kde & $309-382$ \\
\hline
\end{tabular}

Concentrations of all primers are shown in Supplementary Table 3. After 1st PCR, gel electrophoresis was performed to check for successful amplification of all targets. For TRB, gel extraction of the specific PCR products was performed prior to the 2nd PCR.

All 1st round PCR products, except TRB PCR products, were diluted 1:50 unless amplicons were very weak. TRB PCR products and PCR products with weak amplicons were used undiluted. Master-mixes for the 2nd PCR and the temperature profiles were identical for all tubes (Table 1). Primers for the 2nd PCR contained sequencing adaptors and sequencing indexes (barcodes). Unique combination of forward and reverse indexes was used for each library. Three microlitres of undiluted TRB PCR products and $1 \mu \mathrm{l}$ of 1:50-diluted IGH, IGK, TRG and TRD PCR products were amplified in the 2nd PCR.

Following 2nd PCR, products from all samples of a run were pooled in equimolar ratios into eight tube-wise subpools and purified by gel extraction (see Table 2 for the amplicon lengths). Finally, the subpools were pooled equimolarly into one final pool. Sequencing was performed on Illumina MiSeq sequencers, using $2 \times 250$ bp v2 chemistry with a final concentration of $7 \mathrm{pM}$ for the amplicon library and $10 \%$ PhiX control added to avoid lowcomplexity library issues. The detailed standard operating procedure is provided as supplementary information.

\section{Bioinformatic protocol}

ARResT/Interrogate [32] was the main bioinformatics platform used in this study. Both Vidjil [37] and IMGT [38] resources are available through ARResT/Interrogate as built-in tools and were employed for specific aspects of this work, mainly analysis of rearrangements with unclear annotation. Data are deposited at EMBL/EBI European Nucleotide Archive (ENA), accession code PRJEB32668.

Demultiplexing was performed accepting no mismatches. Reads were annotated with EuroClonality-NGS primer sequences (to trim non-amplicon sequences, and for the cPT-QC-based quality control), paired-end joined, dereplicated, immunogenetically annotated [39], and eventually classified into rearrangement types (complete and incomplete, and other special types like intron-Kde rearrangements), or 'junction classes'. Reads without rearrangement were excluded from the total read count used for relative abundances.

cIT-QC sequences described above and elsewhere (see accompanying manuscript by Knecht et al. [30]), were identified in the data through their immunogenetic annotation. Their counts served both as 'in-tube' control and for normalization per primer set: total cIT-QC cells are divided by cIT-QC total reads, the resulting factor used to convert rearrangement reads to cells, and those cells then further divided by total input cells (15,000 in this study). Identified IG/TR sequences were defined as index sequences if their abundance after cIT-QC normalisation exceeded 5\%.

ARResT/Interrogate can track the DNJ $3^{\prime}$ stem of a junction, the sequence remaining stable during IGH or TRB clonal evolution in case of $\mathrm{V}$ replacement or ongoing $\mathrm{V}$ to DJ rearrangements. The stem consists of the last $\leq 3 n t$ of $D$ (or of the NDN if no D is identifiable), any and all of N2 nucleotides, and the $\mathrm{J}$ nucleotides of the junction. This stem is available as a separate immunogenetic feature across all samples and thus can be linked to other features, e.g. clonotypes.

\section{Multicentre validation of assays for MRD marker identification in ALL}

Next, 50 ALL diagnostic samples (29 BCP-ALL and 21 TALL; Supplementary Table 4) were analysed for the multicentre validation study. Each of the five participating laboratories received preconfigured 96-well plates containing the different multiplexed NGS primer combinations per target (Fig. 2).

In total, 96 libraries were generated per lab (total of 480 libraries), and sequenced with a collective output of $47 \mathrm{M}$ reads ( $\varnothing 9.2 \mathrm{M} / \mathrm{lab}$ ). Centralised analysis was performed with ARResT/Interrogate [32] using IMGT germline sequences [39] — further analyses and verifications were performed with Vidjil [37] and IMGT/V-QUEST [38].

Overall, 311 clonal IG/TR rearrangements (clonotypes) were identified, with a mean of $5.2(0-14) /$ sample by NGS (a $5 \%$ threshold was applied for NGS after cIT-QC-based normalization) vs. $5.0(0-14) /$ sample by Sanger, while 217 (45\%) libraries demonstrated no clonotypes above threshold by either method. A total of 196/311 (63\%) clonotypes were fully concordant between NGS and Sanger (Fig. 3). NGS exclusively identified 63/311 (20\%) index sequences, whereas $52 / 311(17 \%)$ IG/TR Sanger sequences were not assigned as NGS index sequence by ARResT/Interrogate. 26/63 NGS positive/Sanger negative cases showed a clonal PCR product 


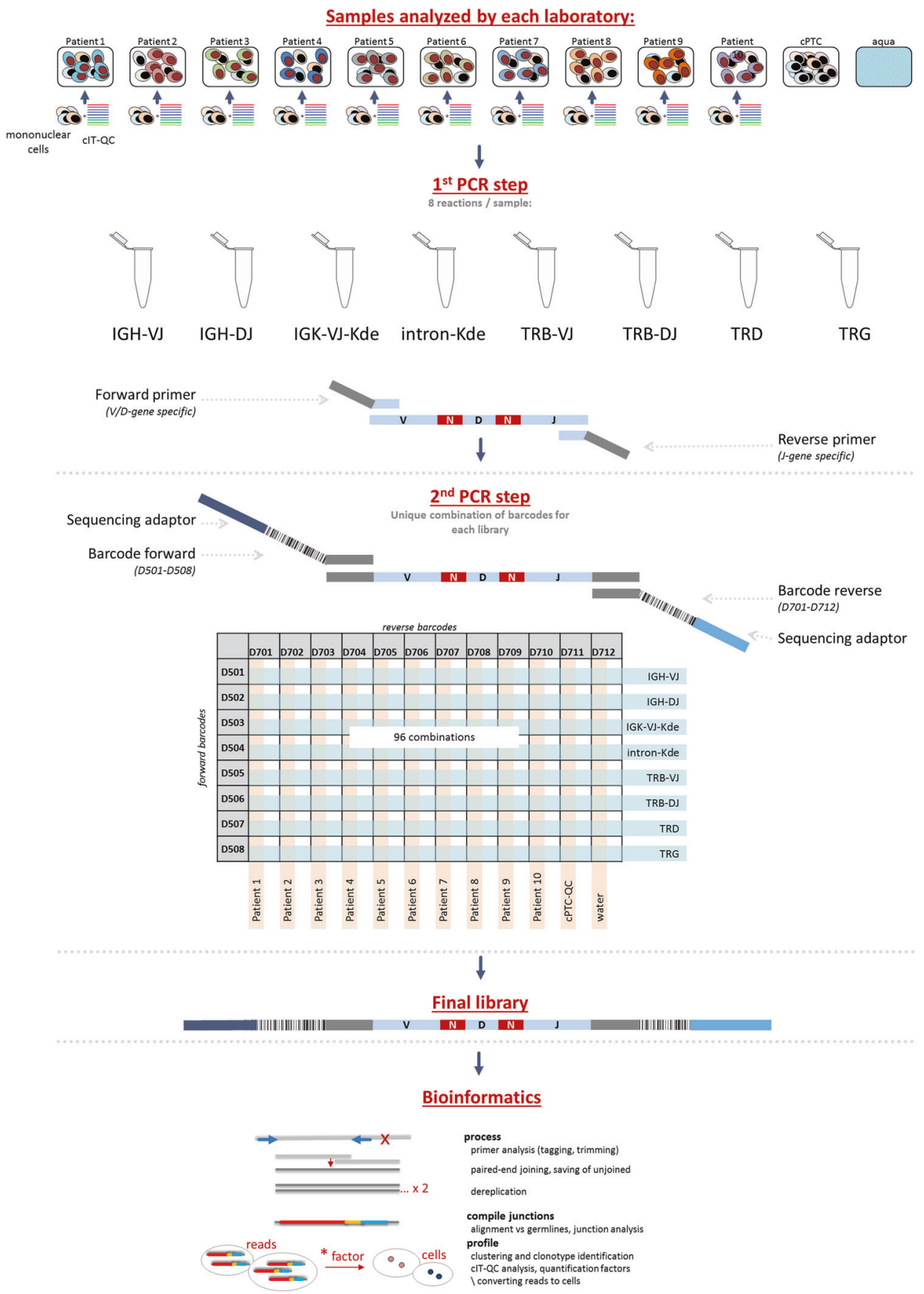

Fig. 2 Schematic overview of the workflow for multicentre validation of IG/TR NGS assays for MRD marker identification in ALL. The IG and TR gene rearrangements are amplified in a two-step approach using multiplex PCR assays. Each of the participating laboratories performed NGS-based IG/TR MRD marker identification in 10

also in the respective low-throughput approach but subsequent Sanger sequencing failed due to polyclonal background, mixed sequences or weak PCR products. In an additional $6 / 63$ NGS positive/Sanger negative cases the respective primer was missing in the low-throughput approach. For the remaining 31/63 discrepancies no patients with ALL. A central polytarget control (cPT-QC) was used to monitor primer performance, and central in-tube controls (cIT-QC) were spiked to each sample as library-specific quality control and calibrator. Pipetting was performed in a 96-well format. The data analysis was performed using ARResT/Interrogate

technical explanation for Sanger failure could be found. In 16/ $19 \mathrm{q} / \mathrm{ddPCR}$ evaluated cases the rearrangement was confirmed by ASO-PCR, in three of these on a subclonal level.

Conversely, 52/311 clonal IG/TR rearrangements were detected by Sanger sequencing only, when applying the 5\% NGS threshold: for 5/52 sequences (1 TRG, 2 TRB-VJ and 


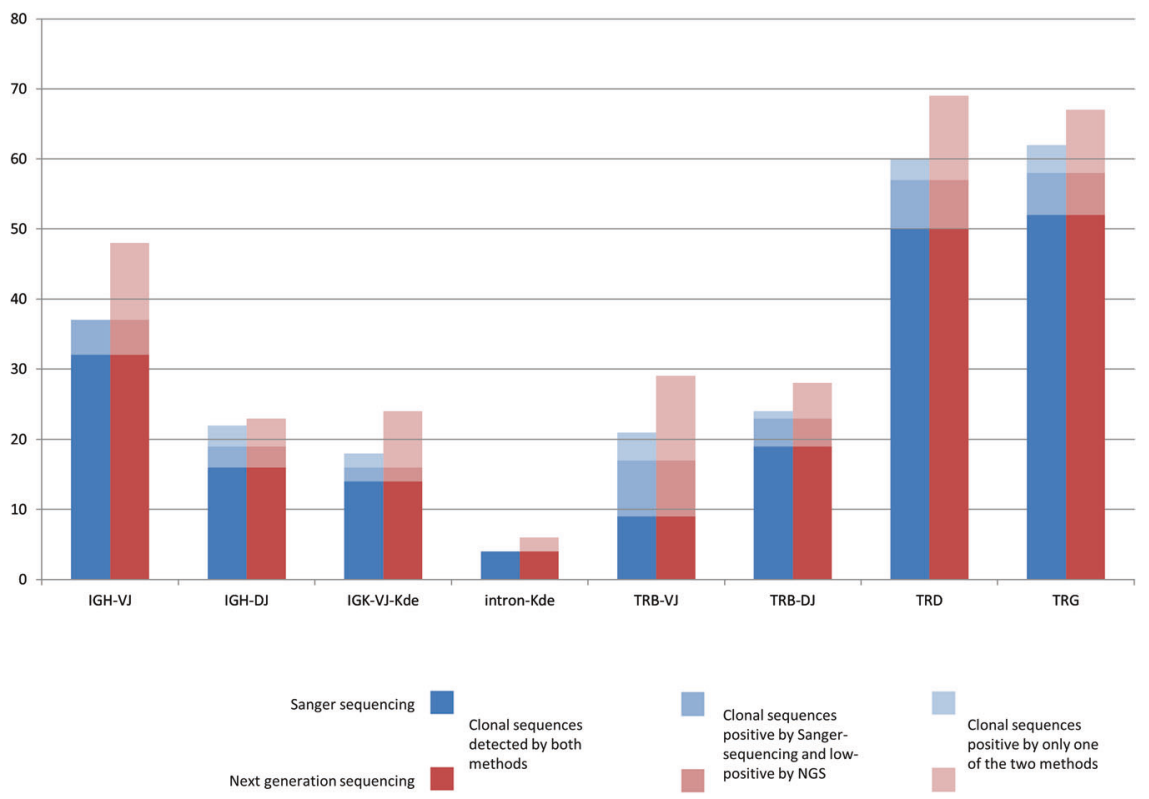

Fig. 3 Results of multicentre validation of assays for MRD marker identification in ALL. Blue: Index sequences identified by Sanger sequencing. Red: Index sequences identified by NGS. Darkest blue/red are clonal sequences identified by both methods; lightest blue/red are

sequences identified only by the respective method. Median blue/red are clonal sequences identified by both methods, but by NGS with an abundance of $<5 \%$ after normalization

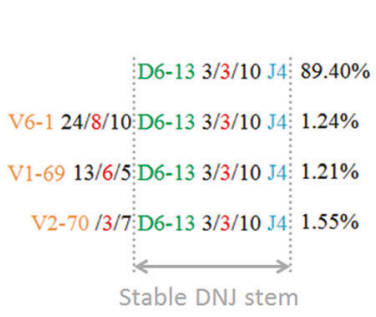

Fig. 4 Clonal evolution in a BCP-ALL patient. The dominant incomplete IGH rearrangement (IGHD6-13 - IGHJ4) was identified with an abundance of $89.4 \%$ together with three additional complete

2 IGH-DJ) the relevant primer was not present in the NGS primer set, in 12/52 cases no explanation was found for the discrepancy. However, in most discordant cases (35/52) the Sanger identified sequences (7 TRD, 8 TRB-VJ, 6 TRG, 4 TRB-DJ, 2 IGK-VJ-Kde, 5 IGH-VJ and 3 IGH-DJ) that were also detectable by NGS, but with an abundance below 5\%. In 36/39 q/ddPCR evaluated cases the rearrangement was confirmed by ASO-PCR (including all low NGS positive sequences), in 14 of these on a subclonal level. The overall concordance between Sanger and NGS, including negative libraries, was $78 \%$.

Interestingly, in 12/29 B-lineage ALL samples the evolution of the dominant clonal IGH sequence was identified employing a special tool in ARResT/Interrogate. The evolved clonotypes shared the DNJ stem with the dominant one, but the VND part of the rearrangement differed (example in Fig. 4).

Assay performance was also analysed by standardized evaluation of QC samples (cIT-QC and cPT-QC, see accompanying manuscript by Knecht et al. [30]) and showed high intra- and inter-lab consistency without statistically significant differences between the five labs.

\section{Modifications of the central SOP}

During the process of multicentre validation, modifications of the SOP were tested in particular laboratories as parallel projects.

\section{One-step versus two-step PCR}

It was decided to use two-step PCR to enable switching of sequencing adaptors and to limit the total number of required primer batches even if a large number of barcodes is required. As first round PCR products are not barcoded, identification of contamination phenomena is hampered in this approach. Therefore, a one-step PCR was tested in a single centre (Paris). The one-step approach reduces the risk of 
contamination and thus favours use of NGS not only for marker identification, but also for MRD assessment. The standard operating procedure is shown in Supplementary information.

\section{Use of Ion Torrent platform}

Ion Torrent platform was tested in a single-centre setting (Prague) and showed a very good concordance $\left(R^{2}=0.89\right)$ with the standard approach. The standard operating procedure is shown in Supplementary information.

\section{Removal of polyclonal DNA from reaction mix}

Polyclonal DNA was added to each reaction in order to prevent excessive primer dimer formation in samples lacking particular rearrangements. The addition of polyclonal DNA, however, alters the composition of polyclonal background of the samples and hampers the analysis of the immune repertoire. We therefore performed testing on four samples with B- and four samples with T-cell aplasia and showed that addition of cIT-QC is sufficient to prevent the excessive formation of unspecific PCR products (see Supplementary information).

\section{Bead extraction}

During the single target evaluation and validation phase, gel extraction of the specific TRB amplicons turned out to lead to more specific libraries compared with bead extraction. However, gel extraction is not used in all laboratories, therefore, in a later phase of the study bead purification of all libraries was also tested. Optimization of the purification processes led to comparable ratios of specific reads irrespective of the type of library purification (Supplementary Table 5).

\section{Discussion}

Amplicon-based IG/TR NGS provides an elegant method to detect clonality, identify MRD markers and monitor MRD in lymphoid malignancies. However, comprehensive SOPs for all relevant IG/TR targets, applicable QC procedures, suitable bioinformatic tools, and validation of the technology in a scientifically controlled, multicentre setting are still lacking [19].

Here we describe an in vitro and in silico protocol for the diagnostic identification of IG/TR MRD markers in ALL, and demonstrate its robustness and applicability across five European laboratories. EuroClonality-NGS primer sets were successfully used with high reproducibility and good concordance to Sanger sequencing, identifying on average $4 \%$ more markers per patient than classical low-throughput methods. NGS was particularly successful in correctly identifying bi-allelic rearrangements, which are technically challenging for Sanger sequencing because this requires prior separation of the respective clonal PCR products. NGS also performs better in the presence of a background of polyclonal rearrangements. Besides, it allows a more comprehensive coverage of rearrangement types. The EuroClonality-NGS TRD assay for example not only detects all types of complete and incomplete TRD gene rearrangements but also VD-JA29 recombinations [40], present in about $20 \%$ of all B-cell precursor (BCP) ALLs. In our current series, these TRDV2-JA29 rearrangements were detected in $7 / 29$ BCP-ALL patients (24\%), providing an attractive target for MRD monitoring. Notably, rearrangement coverage is not complete. The IGH-DJ tube lacks an IGHD7 primer because that would predominantly amplify the germline-configured IGH-IGHD7-IGHJ1.

Low-throughput sequencing of clonal IG/TR gene rearrangements is often cumbersome. This particularly holds true for TRB, where Sanger sequencing of clonal TRB BIOMED-2 amplicons requires a multistep approach: first with the complete set of primers to identify the rearranged genes, and second, a repetition of the sequencing reaction with gene-specific primers. In contrast, the EuroClonalityNGS assays do not require specific workflows for particular targets, thus enormously streamlining the process of MRD marker identification. This becomes increasingly important in times of MRD-based treatment requiring early patient assignment to the respective MRD risk group.

Critically, our assays provide ways to evaluate primer performance and overall quality of the whole NGS run (primers in the cPT-QC) and of each tube (spike-ins in the cIT-QC, see accompanying manuscript by Knecht et al. [30]). Such functionalities are embedded in the ARResT/ Interrogate pipeline, further standardizing the whole workflow. A challenge for correct MRD marker identification in NGS data is the phenomenon of accompanying lymphoid clones that might be mixed up with the leukaemia-specific ones. Therefore, information regarding blast infiltration of the analysed sample must be related to the combined abundance information of the clonal rearrangement, the polyclonal background and the cIT-QC sequences. The integration of all this information allows for a more specific assignment compared with published approaches that define an index sequence simply as sequence with an abundance of $>5 \%$ [16]. This is particularly necessary for tubes that exclusively cover rearrangements being present only in a minority of lymphoid cells (especially the TRD and intronKde tubes). TRD genes are not rearranged in normal B cells and are deleted in most $\mathrm{TR} \gamma \delta$ cells [41]. Therefore, oligoclonal TCR $\gamma \delta \mathrm{T}$ cells might give rise to dominant clonotypes in TRD NGS assay, in particular as the normal TCR $\gamma \delta$ T-cell repertoire is strikingly skewed during childhood. 
Here the cIT-QC-based abundance correction is of utmost importance to avoid miss-assignment of (minor) clonal TRD rearrangements from minor TCR $\gamma \delta$ cell populations as leukaemic rearrangements. Also, knowledge on rearrangement patterns in ALL is important. BCP-ALL features neither complete TRD, nor TRBJ1 gene rearrangements, TALL in contrast generally does not harbour complete IGH or IGK gene rearrangements [42]. Hence, identification of such rearrangements would actually reflect more the presence of accompanying T- and B-cell clones, respectively. This immunogenetic knowledge is of particular importance if marker identification is performed, e.g. at relapse after stem cell transplantation, when patients often harbour a restricted B- and T-cell repertoire. The EuroClonality-NGS approach allows for the bioinformatic identification and correction of this phenomenon, whereas conventional lowthroughput approaches do not harbour correction mechanisms. Nevertheless, we urge caution in assignment of minor clones to the ALL. Although smaller subclones might be missed based on an abundance threshold (which largely explains discrepancies between Sanger sequencing and NGS in our study), decreasing the threshold would be at the expense of specificity.

Oligoclonality is a well-known phenomenon in ALL that hampers conventional IG/TR MRD [43] assessment, but this can be better identified by NGS. Multiple IG/TR gene rearrangements in ALL result from both continuing rearrangement processes (e.g. continuing IGHV to DJ joining) and from secondary rearrangements (e.g. IGH-DJ replacements, $\mathrm{V}$ replacement in a complete IGH rearrangement) [23, 44-49]. In 12 of 29 (41.4\%) patients with B-lineage ALL, a dominant clonal IGH rearrangement was subjected to clonal evolution, resulting in the presence of smaller subclones with the same D-J stem, but different V-genes. D$\mathrm{J}$ replacements are also an evolutionary possibility but cannot be unambiguously discriminated from unrelated lymphoid clones even with sophisticated bioinformatic tools.

Modifications to the here described EuroClonality-NGS assays would be possible, and have actually been tested and approved to be suitable within the working group. In particular, a one-step instead of the two-step PCR presented here might be a reasonable alternative for sites that would apply NGS not only for marker identification but also for MRD assessment. Finally, the Ion Torrent platform was successfully tested as a replacement for the Illumina MiSeq used in this study, and has subsequently also been applied more extensively for clonality assessment in formalin-fixed paraffin-embedded tissue (see accompanying manuscript by Scheijen et al. [31]).

In summary, the EuroClonality-NGS developed an IG/ TR marker identification protocol, which was validated across many expert European laboratories. It covers in vitro and in silico requirements and allows for quality-controlled, streamlined, comprehensive detection of clonal IG/TR rearrangements in ALL. Compared with low-throughput methods, more MRD markers are identified, sensitivity is increased, processing time is reduced and labour-intensive conventional methods to resolve mixed sequences in case of bi-allelic rearrangements or background are avoided. In parallel, the ARResT/Interrogate bioinformatic platform has been developed with specific functionalities addressing potential pitfalls of IG/TR marker identification in ALL, thus enabling a standardized workflow. In addition, the presented approach forms the basis for future applications in clonality assessment, repertoire analysis and MRD quantification in a quality-controlled and accreditable assay with the potential to meet the upcoming European criteria (EU Regulation 2017/746) for in vitro diagnostics.

Acknowledgements Analyses in Brno were supported by Ministry of Health of the Czech Republic, grant no. 16-34272A. Computational resources by the CESNET LM2015042 and the CERIT Scientific Cloud LM2015085, under the programme "Projects of Large Research, Development, and Innovations Infrastructures". Analyses in the Monza (Centro Ricerca Tettamanti, SS, AG, GF and GC) laboratory were supported by the Italian Association for Cancer Research (AIRC) and Comitato Maria Letizia Verga. Analyses in the Paris (Necker, AP-HP) laboratory were supported by the Ile de France CancéroPôle. Analyses in Prague were supported by AZV 16-32568A, and PRIMUS/17/MED/11. Design of IGK assays and analyses in Nijmegen and Rotterdam laboratories were supported by an Innovation project granted by the Zorgverzekeraars Nederland (number 20173442). This publication presents independent research commissioned by the Health Innovation Challenge Fund R9-486, a parallel funding partnership between the Department of Health \& Social Care and Wellcome Trust. The views expressed in this publication are those of the author(s) and not necessarily those of the Department of Health \& Social Care or Wellcome Trust.

\section{Compliance with ethical standards}

Conflict of interest The EuroClonality-NGS Working Group is an independent scientific subdivision of EuroClonality that aims at innovation, standardization and education in the field of diagnostic clonality analysis. The revenues of the previously obtained patent (PCT/NL2003/000690), which is collectively owned by the EuroClonality Foundation and licensed to InVivoScribe, are exclusively used for EuroClonality activities, such as for covering costs of the Working Group meetings, collective WorkPackages and the EuroClonality Educational Workshops. The EuroClonality consortium operates under an umbrella of ESLHO, which is an official EHA Scientific Working Group. MB: contract research for Affimed, Amgen, Regeneron, advisory board of Amgen, Incyte, Speaker bureau of Janssen, Pfizer, Roche. AWL: contract research for Roche-Genentech, research support from Gilead, advisory board for AbbVie, speaker for Gilead, Janssen. RG-S: research grants from Gilead, Takeda, Amgen, and the Spanish government; and reports consulting fees from Janssen, Takeda, Incyte, and BMS. KS: research support from Janssen, Abbvie, Gilead; speaker for Janssen, Abbvie, Gilead; advisory board for Janssen, Abbvie, Gilead. PG: speaker for Gilead.

Publisher's note: Springer Nature remains neutral with regard to jurisdictional claims in published maps and institutional affiliations. 
Open Access This article is licensed under a Creative Commons Attribution 4.0 International License, which permits use, sharing, adaptation, distribution and reproduction in any medium or format, as long as you give appropriate credit to the original author(s) and the source, provide a link to the Creative Commons license, and indicate if changes were made. The images or other third party material in this article are included in the article's Creative Commons license, unless indicated otherwise in a credit line to the material. If material is not included in the article's Creative Commons license and your intended use is not permitted by statutory regulation or exceeds the permitted use, you will need to obtain permission directly from the copyright holder. To view a copy of this license, visit http://creativecommons. org/licenses/by/4.0/.

\section{References}

1. Tonegawa S. Somatic generation of antibody diversity. Nature. 1983;302:575-81.

2. Davis MM, Bjorkman PJ. T-cell antigen receptor genes and T-cell recognition. Nature. 1988;334:395-402.

3. Schlissel MS. Regulating antigen-receptor gene assembly. Nat Rev Immunol. 2003;3:890-9.

4. Lefranc M-P, Lefranc G. The T cell receptor factsbook. Academic Press; 2001. https://www.sciencedirect.com/science/ book/9780124413528. Accessed 22 Mar 2018.

5. Lefranc M-P, Lefranc G The immunoglobulin factsbook. Academic Press; 2001

6. Monroe JG, Dorshkind K. Fate decisions regulating bone marrow and peripheral B lymphocyte development. Adv Immunol. 2007;95:1-50.

7. von Boehmer H, Melchers F. Checkpoints in lymphocyte development and autoimmune disease. Nat Immunol. 2010;11:14-20.

8. Evans PAS, Pott C, Groenen PJTA, Salles G, Davi F, Berger F, et al. Significantly improved PCR-based clonality testing in B-cell malignancies by use of multiple immunoglobulin gene targets. Report of the BIOMED-2 Concerted Action BHM4-CT98-3936. Leukemia. 2007;21:207-14.

9. Brüggemann M, White H, Gaulard P, Garcia-Sanz R, Gameiro P, Oeschger $S$, et al. Powerful strategy for polymerase chain reaction-based clonality assessment in T-cell malignancies Report of the BIOMED-2 Concerted Action BHM4 CT98-3936. Leukemia. 2007;21:215-21.

10. Langerak AW, Groenen PJTA, Brüggemann M, Beldjord K, Bellan C, Bonello L, et al. EuroClonality/BIOMED-2 guidelines for interpretation and reporting of $\mathrm{Ig} / \mathrm{TCR}$ clonality testing in suspected lymphoproliferations. Leukemia. 2012;26:2159-71.

11. van Dongen JJM, Langerak AW, Brüggemann M, Evans PAS, Hummel M, Lavender FL, et al. Design and standardization of PCR primers and protocols for detection of clonal immunoglobulin and T-cell receptor gene recombinations in suspect lymphoproliferations: report of the BIOMED-2 Concerted Action BMH4-CT98-3936. Leukemia. 2003;17:2257-317.

12. Boyd SD, Marshall EL, Merker JD, Maniar JM, Zhang LN, Sahaf $\mathrm{B}$, et al. Measurement and clinical monitoring of human lymphocyte clonality by massively parallel VDJ pyrosequencing. Sci Transl Med. 2009;1:12ra23.

13. DeKosky BJ, Ippolito GC, Deschner RP, Lavinder JJ, Wine Y, Rawlings BM, et al. High-throughput sequencing of the paired human immunoglobulin heavy and light chain repertoire. Nat Biotechnol. 2013;31:166-9.

14. Bartram J, Goulden N, Wright G, Adams S, Brooks T, Edwards $\mathrm{D}$, et al. High throughput sequencing in acute lymphoblastic leukemia reveals clonal architecture of central nervous system and bone marrow compartments. Haematologica. 2018;103: e110-e114.
15. Faham M, Zheng J, Moorhead M, Carlton VE, Stow P, CoustanSmith E, et al. Deep-sequencing approach for minimal residual disease detection in acute lymphoblastic leukemia. Blood. 2012;120:5173-80.

16. Ladetto M, Bruggemann M, Monitillo L, Ferrero S, Pepin F, Drandi $\mathrm{D}$, et al. Next-generation sequencing and real-time quantitative PCR for minimal residual disease detection in B-cell disorders. Leukemia. 2014;28:1299-307.

17. Pulsipher MA, Carlson C, Langholz B, Wall DA, Schultz KR, Bunin N, et al. IgH-V(D)J NGS-MRD measurement pre- and early post- allo-transplant defines very low and very high risk ALL patients. Blood. 2015;125:3501-8.

18. Kotrova $M$, Muzikova $\mathrm{K}$, Mejstrikova $\mathrm{E}$, Novakova $\mathrm{M}$, Bakardjieva-Mihaylova V, Fiser K, et al. The predictive strength of next-generation sequencing MRD detection for relapse compared with current methods in childhood ALL. Blood. 2015;126:1045-7.

19. Langerak AW, Brüggemann M, Davi F, Darzentas N, Gonzalez $D$, Cazzaniga $G$, et al. High throughput immunogenetics for clinical and research applications in immunohematology: potential and challenges. J Immunol. 2017;198:3765-74.

20. Kotrova M, van der Velden VHJ, van Dongen JJM, Formankova $\mathrm{R}$, Sedlacek P, Brüggemann M, et al. Next-generation sequencing indicates false-positive MRD results and better predicts prognosis after SCT in patients with childhood ALL. Bone Marrow Transpl. 2017;52:962-8.

21. Kotrova M, Trka J, Kneba M, Brüggemann M. Is next-generation sequencing the way to go for residual disease monitoring in acute lymphoblastic leukemia? Mol Diagn Ther. 2017. https://doi.org/ 10.1007/s40291-017-0277-9.

22. Freeman JD, Warren RL, Webb JR, Nelson BH, Holt RA. Profiling the T-cell receptor beta-chain repertoire by massively parallel sequencing. Genome Res. 2009;19:1817-24.

23. Gawad C, Pepin F, Carlton VEH, Klinger M, Logan AC, Miklos $\mathrm{DB}$, et al. Massive evolution of the immunoglobulin heavy chain locus in children with B precursor acute lymphoblastic leukemia. Blood. 2012;120:4407-17.

24. Logan AC, Gao H, Wang C, Sahaf B, Jones CD, Marshall EL, et al. High-throughput VDJ sequencing for quantification of minimal residual disease in chronic lymphocytic leukemia and immune reconstitution assessment. Proc Natl Acad Sci USA. 2011;108:21194-9.

25. Logan AC, Zhang B, Narasimhan B, Carlton V, Zheng J, Moorhead $\mathrm{M}$, et al. Minimal residual disease quantification using consensus primers and high-throughput IGH sequencing predicts posttransplant relapse in chronic lymphocytic leukemia. Leukemia. 2013;27:1659-65.

26. Robins HS, Srivastava SK, Campregher PV, Turtle CJ, Andriesen J, Riddell SR, et al. Overlap and effective size of the human $\mathrm{CD} 8+\mathrm{T}$ cell receptor repertoire. Sci Transl Med. 2010;2:47ra64-47ra64.

27. Wang C, Sanders CM, Yang Q, Schroeder HW, Wang E, Babrzadeh $\mathrm{F}$, et al. High throughput sequencing reveals a complex pattern of dynamic interrelationships among human $\mathrm{T}$ cell subsets. Proc Natl Acad Sci. 2010;107:1518-23.

28. Wu D, Sherwood A, Fromm JR, Winter SS, Dunsmore KP, Loh ML, et al. High-throughput sequencing detects minimal residual disease in acute $\mathrm{T}$ lymphoblastic leukemia. Sci Transl Med. 2012;4:134ra63-134ra63.

29. Wu Y-C, Kipling D, Leong HS, Martin V, Ademokun AA, DunnWalters DK. High-throughput immunoglobulin repertoire analysis distinguishes between human IgM memory and switched memory B-cell populations. Blood. 2010;116:1070-8.

30. Knecht H, Reigl T, Kotrová M, Appelt F, Stewart P, Bystry V, et al. Quality control and quantification in IG/TR next-generation sequencing marker identification: protocols and bioinformatic 
functionalities by EuroClonality-NGS. Leukemia; revision. [Epub ahead of print]

31. Scheijen B, Meijers R, Rijntjes J, van der Klift M, Möbs M, Steinhilber $\mathrm{J}$, et al. Next-generation sequencing of immunoglobulin gene rearrangements for clonality assessment: a technical feasibility study by EuroClonality-NGS. Leukemia; revision. [Epub ahead of print]

32. Bystry V, Reigl T, Krejci A, Demko M, Hanakova B, Grioni A, et al. ARResT/Interrogate: an interactive immunoprofiler for IG/ TR NGS data. Bioinformatics. 2016;33:btw634.

33. Rozen S, Skaletsky H. Primer3 on the WWW for general users and for biologist programmers. Methods Mol Biol. 2000;132:365-86.

34. Qu W, Zhou Y, Zhang Y, Lu Y, Wang X, Zhao D, et al. MFEprimer-2.0: a fast thermodynamics-based program for checking PCR primer specificity. Nucleic Acids Res. 2012;40:W205-8.

35. van der Velden VHJ, Cazzaniga G, Schrauder A, Hancock J, Bader P, Panzer-Grumayer ER, et al. Analysis of minimal residual disease by $\mathrm{Ig} / \mathrm{TCR}$ gene rearrangements: guidelines for interpretation of real-time quantitative PCR data. Leukemia. 2007;21:604-11.

36. Pongers-Willemse MJ, Seriu T, Stolz F, D’Aniello E, Gameiro P, Pisa $\mathrm{P}$, et al. Primers and protocols for standardized detection of minimal residual disease in acute lymphoblastic leukemia using immunoglobulin and $\mathrm{T}$ cell receptor gene rearrangements and TAL1 deletions as PCR targets: report of the BIOMED-1 CONCERTED ACTION. Leukemia. 1999;13:110-8.

37. Duez M, Giraud M, Herbert R, Rocher T, Salson M, Thonier F, et al. Vidjil: a web platform for analysis of high-throughput repertoire sequencing. PLoS ONE. 2016;11:e0166126.

38. Giudicelli V, Brochet X, Lefranc M-P. IMGT/V-QUEST: IMGT standardized analysis of the immunoglobulin (IG) and $\mathrm{T}$ cell. Recept (TR) Nucleotide Seq Cold Spring Harb Protoc. 2011;2011:695-715.

39. Giudicelli V, Chaume D, Lefranc MP. IMGT/GENE-DB: a comprehensive database for human and mouse immunoglobulin and T cell receptor genes. Nucleic Acids Res. 2005;33:D256-61.

40. Szczepanski T, Van Der Velden VHJ, Hoogeveen PG, De Bie M, Jacobs CH, Van Wering ER, et al. Vdelta2-Jalpha rearrangements are frequent in precursor-B-acute lymphoblastic leukemia but rare in normal lymphoid cells. Blood. 2004;103:3798-804.

41. Lefranc MP, Rabbitts TH. Genetic organization of the human T-cell receptor gamma and delta loci. Res Immunol. 1990;141:565-77.

42. Dongen J van, Szczepanski T, Adriaansen H. Immunobiology of leukemia. 7th edn. WB Saudners Company: Philadelphia; 2002.

43. van Dongen JJ, Seriu T, Panzer-Grumayer ER, Biondi A, Pongers-Willemse MJ, Corral L, et al. Prognostic value of minimal residual disease in acute lymphoblastic leukaemia in childhood. Lancet. 1998;352:1731-8.

44. Kitchingman GR. Immunoglobulin heavy chain gene VH-D junctional diversity at diagnosis in patients with acute lymphoblastic leukemia. Blood. 1993;81:775-82.

45. Steenbergen EJ, Verhagen OJ, van Leeuwen EF, von dem Borne $\mathrm{AE}$, van der Schoot CE. Distinct ongoing Ig heavy chain rearrangement processes in childhood B-precursor acute lymphoblastic leukemia. Blood. 1993;82:581-9.

46. Szczepański T, Willemse MJ, Brinkhof B, van Wering ER, van der Burg M, van Dongen JJM. Comparative analysis of Ig and TCR gene rearrangements at diagnosis and at relapse of childhood precursor-B-ALL provides improved strategies for selection of stable PCR targets for monitoring of minimal residual disease. Blood. 2002;99:2315-23.

47. de Haas V, Verhagen OJ, von dem Borne AE, Kroes W, van den Berg H, van der Schoot CE. Quantification of minimal residual disease in children with oligoclonal B-precursor acute lymphoblastic leukemia indicates that the clones that grow out during relapse already have the slowest rate of reduction during induction therapy. Leukemia. 2001;15:134-40.

48. Germano G, del Giudice L, Palatron S, Giarin E, Cazzaniga G, Biondi A, et al. Clonality profile in relapsed precursor-B-ALL children by GeneScan and sequencing analyses. Consequences on minimal residual disease monitoring. Leukemia. 2003;17:1573-82.

49. Theunissen PMJ, van Zessen D, Stubbs AP, Faham M, Zwaan $\mathrm{CM}$, van Dongen JJM, et al. Antigen receptor sequencing of paired bone marrow samples shows homogeneous distribution of acute lymphoblastic leukemia subclones. Haematologica. 2017;102:1869-77.

\title{
Affiliations
}

\begin{abstract}
Monika Brüggemann ${ }^{1} \cdot$ Michaela Kotrová ${ }^{1,2} \cdot$ Henrik Knecht $^{1} \cdot$ Jack Bartram $^{3} \cdot$ Myriam Boudjogrha $^{4} \cdot$ Vojtech Bystry ${ }^{5}$ - Grazia Fazio $\mathbb{D}^{6} \cdot$ Eva Froňková ${ }^{2} \cdot$ Mathieu Giraud $\mathbb{D}^{7}$ - Andrea Grioni ${ }^{6} \cdot$ Jeremy Hancock $^{8}$. Dietrich Herrmann ${ }^{1} \cdot$ Cristina Jiménez $^{9} \cdot$ Adam Krejci $^{5} \cdot$ John Moppett $^{10} \cdot$ Tomas Reigl $^{5} \cdot$ Mikael Salson $^{7}$. Blanca Scheijen ${ }^{11} \cdot$ Martin Schwarz $^{1}$ - Simona Songia ${ }^{6}$ - Michael Svaton ${ }^{2}$ - Jacques J. M. van Dongen ${ }^{12}$. Patrick Villarese $^{13}$ - Stephanie Wakeman ${ }^{8}$ - Gary Wright ${ }^{3} \cdot$ Giovanni Cazzaniga $^{6}$ - Frédéric Davi ${ }^{4}$.
\end{abstract}

Ramón García-Sanz ${ }^{9}$ - David Gonzalez ${ }^{14}$ - Patricia J. T. A. Groenen ${ }^{11}$ - Michael Hummel ${ }^{15}$ - Elizabeth A. Macintyre ${ }^{13}$.

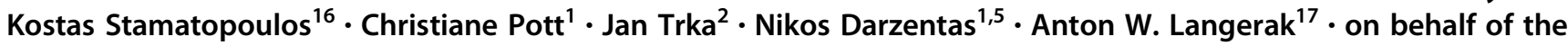
EuroClonality-NGS working group

1 Department of Hematology, University Hospital SchleswigHolstein, Kiel, Germany

2 CLIP - Childhood Leukaemia Investigation Prague, Department of Paediatric Haematology and Oncology, Second Faculty of Medicine, Charles University, University Hospital Motol, Prague, Czech Republic

3 Department of Paediatric Haematology, Great Ormond Street Hospital, London, UK

4 Department of Hematology, Hopital Pitié-Salpêtrière,
Paris, France

5 Central European Institute of Technology, Masaryk University, Brno, Czech Republic

6 Centro Ricerca Tettamanti, University of Milano Bicocca, Monza, Italy

7 CNRS, CRIStAL, Université Lille, Inria Lille, France

8 Bristol Genetics Laboratory, Southmead Hospital, Bristol, UK

9 Hospital Universitario de Salamanca-IBSAL, Salamanca, Spain 
10 Department of Pediatric Haematology, Bristol Royal Hospital for Children, Bristol, UK

11 Department of Pathology, Radboud University Medical Center, Nijmegen, The Netherlands

12 Department of Immunohematology and Blood Transfusion (IHB), Leiden University Medical Center, Leiden, The Netherlands

13 Department of Hematology, APHP Necker-Enfants Malades and Paris Descartes University, Paris, France
14 Centre for Cancer Research and Cell Biology, Queen's University Belfast, Belfast, UK

15 Insititute of Pathology, Charité - Universitätsmedizin Berlin, Berlin, Germany

16 Institute of Applied Biosciences, Thessaloniki, Greece

17 Department of Immunology, Laboratory Medical Immunology, Erasmus MC, University Medical Center, Rotterdam, The Netherlands 